\title{
Die Produktivität eines Phantoms
}

\section{Lichtenberg, das Phlogiston und die neue Chemie Lavoisiers [1988/1994]}

\author{
Lichtenberg schreibt am 20. Mai 1782 an Wolff:
}

Ich habe seit einiger Zeit die Elektrizität etwas liegen lassen und beschäftige mich dafür mit den herrlichen Erscheinungen, welche die Entdeckungen mit den Luftarten gewähren. Ich habe in diesen Tagen eine Sackuhrfeder, die über einen Fuß lang war, in dephlogistisierter Luft angesteckt, daß sie abbrannte wie ein Bindfaden und das mit einem Licht, daß man auf 10 Schritte davon einen feinen Druck lesen konnte.

Kurz darauf gelingt es ihm, eine Uhrfeder und eine englische Feldmesserklinge bei der gleichen Flamme zu verschmelzen; er sendet die Trophäe Wolff. Zwischen Göttingen und Hannover gehen gefüllte und leere Flaschen mit dephlogistisierter Luft hin und her wie die Zieheimer in einem Brunnen. Die Studenten schwänzen nicht mehr, seit es blitzt und donnert.

Aus Darmstadt erreicht mich per Eilpost weder Phlogiston noch dephlogistisierte Luft, und doch ein entzündlicher Stoff, ein Auszug aus dem Register der Lichtenberg-Ausgabe des Hanser Verlags, ${ }^{1}$ der Hinweis auf so merkwürdige Stellen wie diese:

Ich glaube man könnte zugeben, daß unter gewissen Umständen aus Wasser dephl. und infl. Luft werden kann, ohne deswegen zuzugeben, daß es daraus zusammengesetzt sei. Denn mein Gott was kann nicht aus Wasser werden. (J 1907)

Bei dem Verbrennen der dephlog. Luft mit infl. Leidenfrosts Versuch mit dem Nagel nachmachen ob man nicht aus Wasser Ruß erhalten kann. (J 1678)

Beim Phlogiston können auch die Erfahrungen vom Geruch der Hunde genützt werden. (J 1904)

Er pflegte zu sagen er müsse seinen gleichsam durch die Glut der Lüste verkalchten Kräften etwas Phlogiston zusetzen (dem Witz Phlogiston zusetzen). (D 316)

Was mich eigentlich bewogen hat, so lange mit meinem Beifall für die antiphlogistische Chemie zurückzuhalten, ist (verzeihe mir meine schwere Sünde,) bloß der enthusiastische Beifall gewesen, womit sie von einigen Leuten beehrt worden ist, deren Flüchtigkeit im Schließen, Seichtigkeit und Ignoranz in der Naturlehre mir bekannt war. (K 336)

1 Dank der Liebenswürdigkeit von Wolfgang Promies. Vorliegende Abhandlung, die der Lichtenberg-Gesellschaft auf ihrer Jahrestagung 1987 präsentiert wurde, wahrt bewusst diesen Vortragston. Zitiert wird nach Lichtenberg $1967 \mathrm{ff}$.

Ә Open Access. (C) Uwe Pörksen, publiziert von De Gruyter. (c) BY-NC-ND Dieses Werk ist lizenziert unter der Creative Commons Attribution-NonCommercial-NoDerivatives 4.0 Lizenz. 


\begin{abstract}
Man tadelt gewöhnlich die Phlogistiker, daß sie unter sich nicht eins sind, der eine dies, der andere das unter Phlogiston versteht, und daß sie folglich selbst nicht einmal wissen was sie wollen. Man zieht daraus sogar ein Argument wider die Existenz des Phlogistons. Ich glaube aber man könnte das Argument grade umkehren und sagen eben deswegen, weil es so vielerlei Meinungen gibt, die doch wenigstens alle darin überein kommen, daß außer Feuer und Luft noch etwas da sein müsse, so ist es sehr wahrscheinlich, daß so etwas da ist. Wenigstens hat man ähnliche Schlüsse aus dem allgemeinen Glauben an einen Gott oder an eine Seele gezogen. (Selbst daß die Menschen Götzen anbeten beweist einen Gott). (J 2134)

Die Lehre von der Seele, ist wie die vom Phlogiston. (J 1306)

Was für große Männer haben sich nicht selbst noch nach Newtons Entdeckungen bemüht die Wirbel des Cartesius zu verteidigen. Dieses Gleichnis ist für die Antiphlogistiker. (J 1701)

Am vernünftigsten ist es, es bei Streitigkeiten so zu machen wie der berühmte Fourcroy, der alle Gegner der franz. Chemie in 2 Klassen bringt, 1) Solche die die Sache nicht verstehen und 2) die die von Parteigeist verleitet werden. (J 1214)
\end{abstract}

\title{
1
}

Die Geschichte der Wissenschaft nimmt gelegentlich die Gestalt eines Parabelmärchens an. Eines dieser parabolischen Märchen könnte den Titel Phlogiston tragen oder Das arme Phlogiston, und es wird vom Beginn des 19. bis ins 20. Jahrhundert etwa so überliefert:

Als der Satz vom Grund, der besagt, dass nichts ohne Grund sei, sein Regiment antrat und man unter anderem auch zu fragen begann, woher es komme, dass etwas brennt - nicht, woher das Feuer stamme, sie kannten ihre Mythologie -, sondern warum etwas in Feuer gerate und nicht eher nicht, als man also nach der Ursache der Brennbarkeit zu fahnden begann, da fand ein Mann das den gesuchten ,Gegenstand' anzielende Wort. Er gab dem Kind einen Namen und taufte es Phlogiston. Natürlich war er nicht der erste, der so fragte und antwortete. Nie ist einer der erste. Van Helmont hatte von dem Sulphur des Paracelsus als dem ,Phlogiston' gesprochen, Ulrich Becher hatte einen entscheidenden Schritt getan und in seiner Physica subterranea, der unterirdischen Physik, aus den tria prima des Paracelsus, aus ,Sulphur', ,Sal', ,Merkur', die drei Erscheinungsformen einer Urerde werden lassen, aus denen alles gemacht sei, terra pinguis, terra fusilis, terra mercurialis, und hatte von der terra pinguis, der fettigen Erde, gesagt, dass sie in allen brennbaren und verkalkbaren Stoffen enthalten sei und beim Erhitzen oder mit der Flamme entweiche (vgl. Lockemann 1950: 89). Auf diese Weise war der Boden bereitet. Georg Ernst Stahl ergriff das „principium der Brennlichkeit“ grundsätzlicher, arbeitete es aus, suchte in ihm den einheitlichen Grund so verschiedener Vorgänge wie der Verkalkung von Metallen und der Verbrennung organischer Substanzen, der Atmung, Gärung und Verwesung. An seinen Namen knüpfte sich so die Theorie und der Name ,Phlogiston‘. 
Es war nicht der einzige Ausdruck, den er gebrauchte, er umschrieb das Gemeinte in einer Reihe von Synonymen, sagte etwa: ,feuerfähiges Principium‘,

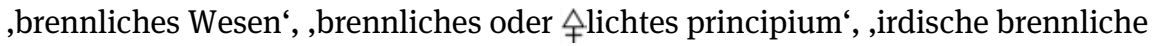
Materie‘, ,principium der Brennlichkeit‘, ,die Brennlichkeit‘, ,principio phlogisto', ,feurigmachende Kraft‘, ,Entzündlichkeit‘, ,das Brennliche‘, ,feuer-fähige Grundmaterie' (vgl. Stahl 1744: 127, 194, 128, 133, 129, 194, 202, 197). Man einigte sich auf

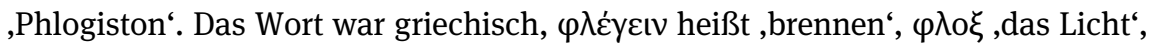
,Phlogiston‘ war ,das Brennbare‘. Die Vorstellung war die, dass in allen brennbaren und in der Hitze veränderlichen Stoffen jenes ,Etwas', das ,Phlogiston“, enthalten sei und dass es bei der Verbrennung entschwinde. Wenn ein Metall veraschte oder, wie man sagte, verkalkte, entwich es; man konnte es in diesem Fall zurückholen, indem man die Metallasche über Kohle erhitzte. Dann wurde der Asche wieder Phlogiston zugeführt und das Metall war zurückgewonnen. Diese „Wiederbelebung“ von Metallen war ein gern wiederholtes Experiment. In reiner Form war der brennbar machende Bestandteil unbekannt. Es war vorerst ein principium und noch keine Materie in späterem Sinn. Das ,Phlogiston‘ Stahls hatte ein langes Leben vor sich, mehr als neunzig Jahre, einen langen Aufstieg und jähen Fall, und ihm war ein in verschiedenerlei Hinsicht peinliches Ende bestimmt: es starb durch Verbrennung.

Das sprachliche Zeichen hat vier Seiten.

1. Zu ihm gehört ein Lautbild; in unserem Fall ist das Wortmaterial griechisch;

2. es bezieht sich auf einen Gegenstand, hier auf das ,principium der Brennlichkeit';

3. es ist von den Gegenständen durch einen Abgrund getrennt, bezieht sich niemals unmittelbar, sondern immer nur mittelbar auf den Gegenstand, auf dem Umweg über Begriffe, Vorstellungen: in unserem Fall auf dem Umweg über die Vorstellung einer ,feurigmachenden Kraft‘ oder ,feuerfähigen Grundmaterie';

4. es erfüllt eine soziale Funktion; z.B. indem es eine Antwort bereithält, eine Art Erklärung gibt auf eine beunruhigende Frage. ,Phlogiston‘ sagt: warum es brennt.

Weil das sprachliche Zeichen jedoch fast unabhängig von der Wirklichkeit, auf die es sich bezieht, dazu gebraucht werden kann, sich mit seiner Hilfe zu verständigen - verschweißt es doch eine bestimmte Vorstellung mit einem fasslichen Laut und versieht als diese Verbindung eine gesellschaftliche Funktion weil dies seine Natur ist, kann es auch in Gebrauch sein, wenn ihm in der Wirklichkeit gar nichts entspricht. Es kann eine imaginäre Zwischenwelt aufbauen, kann sie auch dann noch fortbestehen lassen, wenn ihr Erfahrungen direkt widersprechen. Fragt sich allerdings, wie lange. 
Der Geist ist ein Wühler, wie es bei Jakob Burckhardt (1949: 8) heißt. Im sprachlichen Zeichen arbeitete an mindestens drei Stellen der Maulwurf:

1. ein Lautbild ist bis $\mathrm{zu}$ einem gewissen Grad arbiträr und lässt sich mit verschiedenen Vorstellungen verbinden; der Laut ,Phlogiston“ war darum am widerstandsfähigsten und hielt sich lange;

2. aber was war der Gegenstand, das ,principium der Brennlichkeit‘, wenn man ihm nachfragte?

3. wie hatte man ihn sich vorzustellen: als Geist, als eine Art Luft, als Materie?

4. konnte man sich auf die Dauer mit einer Antwort zufrieden geben, die, wie Schelling rückblickend feststellte, auf die Frage „Was macht die Körper brennbar?“, erwiderte „Dasjenige, was sie brennbar macht?“(Färber 1927:124) - Und war nicht das Prinzip der personalen und nationalen Konkurrenz der Wissenschaft längst eingebaut und ein unaufhaltsamer Motor wider den Stillstand?

Vorerst lebte das Phlogiston, fand Anhänger in Deutschland, England, Frankreich, wurde auf Kathedern weitergereicht, ja, man kann sagen, es materialisierte sich. Insbesondere auf seiner Wanderung nach Frankreich gewann es an Stofflichkeit. Öl, so schien es, enthielt viel Phlogiston, der Kohlenstoff kam ihm sehr nahe. Es wurde mit einem Symbol versehen. Der Schwede Torbern Bergman gab ihm das Zeichen eines Dreiecks (= Materie der Wärme) mit schleifenförmigen Winkeln und einem Kreuzchen (= Säure) unter der Grundseite: $\frac{1}{\mp}$ (Walden 1927: 87; vgl. Cordier 1928: 40, 43).

Es war die Frage seines Gewichts, was den Keil des Zweifels in die Brust der Wissensdurstigen trieb. Metalle, die in der Hitze veraschen und denen also das Phlogiston entweicht, sind nachher merklich schwerer. Blei oder Quecksilber z. B. nehmen, wenn sie verkalken, an Gewicht zu. Wieso werden sie schwerer, wenn ihnen etwas verloren geht, und leichter, wenn ihnen das Phlogiston wieder zugeführt wird?

Stahl war dieser Sachverhalt bekannt, aber er hatte ihn nicht beschäftigt, ein sicheres Zeichen, dass Phlogiston für ihn ein principium im Paracelsischen Sinn, eine qualitas war und kein als Quantität zu fassender Stoff. Nicht um die materia, sondern um die Wirkung auf die materia (actiones in materias) war es ihm zu tun (Driesch 1922: 31).

Einige Schüler beunruhigte aber die Frage, und sie fanden verschiedene Erklärungen. Sie nahmen z.B. an, dass Phlogiston spezifisch leichter sei als die Luft, in welcher die Wägungen ausgeführt wurden, das leichte Phlogiston vermindere das Gewicht des Metalls, so wie der Korken an der Angelschnur das daranhängende Blei zum Schwimmen bringt. Phlogiston erschien geradezu als ein Stoff von ,negativer Schwere' oder ,absoluter und positiver Leichtigkeit': eben deshalb wurde das Metall bei seinem Entschwinden schwerer (Färber 1927: 124; Lockemann 
1950: 130f.). Hatte nicht schon Aristoteles die Erde als Prinzip des Schweren und das Feuer als das des nach oben strebenden Leichten bezeichnet? - Je mehr man geneigt war, die Gewichtsverhältnisse als etwas wirklich Bedeutsames zu werten, von einer qualitativen Chemie zu einer quantitativen Chemie hinüber zu wechseln, um so geringer wurden die Überlebenschancen des Phlogiston.

Der Geist wühlte weiter. Worin besteht das Wesen der Verkalkung und der Atmung? Das antike Element Luft stand längst im Verdacht, von zusammengesetzter Natur zu sein. Im Frühjahr 1774 fand der Franzose Pierre Bayen, als er rotes Quecksilberoxyd - ein Metallkalk - erhitzte, dass es sich in Quecksilber zurückverwandelte und dabei nicht etwas aufnahm aus der Luft, sondern ein Gas abgab. Er hielt den Schlüssel in der Hand, so wird berichtet, aber er verstand das Versuchsergebnis nicht. Hätte er nur einen kleinen glimmenden Holzspan in sein „fluide elastique“ gehalten, so würde er statt Priestley als Entdecker des Sauerstoffs gefeiert worden sein (Lockemann 1950: 134).

Priestley stellte am 1. August 1774, als er ebenfalls rotes Quecksilberoxyd erhitzte, das gleiche Gas dar und erkannte, dass es die Verbrennung begünstigte. Es schien, als zöge es das Phlogiston an. Priestley beharrte auf der Vorstellung, dass bei Verbrennungen Phlogiston abgegeben wird. Das neu entdeckte Gas zog das Phlogiston offenbar darum an, weil es selbst keines enthielt. Was später ,Sauerstoff‘ hieß, nannte er deshalb ,dephlogistisierte Luft‘ (Dannemann 1903: 305). Der andere Bestandteil dagegen, in dem nichts mehr brennt und der Mensch nicht atmen kann - also die 4/5 der Atmosphäre, die wir "Stickstoff‘ nennen erschien ihm als ganz und gar von Phlogiston gesättigte Luft. Er nannte diese Luft, die keinen ,Brennstoff‘ mehr aufnahm, ,phlogistische Luft‘. Hätte Priestley nicht den Widerspruch bemerken müssen, fragt ein Überlieferer, der darin liegt, dass die Luft bei der Verbrennung an Volumen und Gewicht verliert statt gesättigt zu werden? Der Geist wühlte weiter. Aber das Phlogiston war eine Institution und das Gewand seiner Sprache wirkte noch fort. Priestleys Augen waren gehalten. Es wurde indes immer schwieriger, am Phlogiston festzuhalten, wie es vor Kepler schwierig wurde, die immer genauer aufgezeichneten Planetenbewegungen auf der Grundlage des ptolemäischen Weltbildes zu beschreiben.

Da erschien Lavoisier und machte dem Spuk ein Ende. Er war nicht der erste; nie ist jemand der erste. Er versuchte es so darzustellen, als habe er alles entdeckt. Aber man kam ihm auf die Schliche. Nicht nur Priestley, auch Scheele hatte vor ihm die ,Feuerluft' und die ,verdorbene Luft“, Stickstoff und Sauerstoff, dargestellt. Lavoisier aber benutzte die Waage und maß das Volumen, und nun konnte der Schleier, der die Wahrheit verhallte, mit einem Mal fallen (Dannemann 1903: 307).

Er zeigte durch Messen und Wiegen unter luftdicht abgeschlossenen Bedingungen, dass bei der Umwandlung eines Metalls in Kalk nicht Phlogiston verloren ging, sondern ein Stoff aufgenommen wurde, der aus der Luft stammte und seit 
Priestley und Scheele als ,phlogistisierte‘ oder ,Feuerluft‘ bekannt war. Und zwar nahm das Metall genau so viel an Gewicht zu, wie die Luft an Gewicht abnahm; und bei dem Umkehrversuch, der durch Glühen erreichten ,Wiederbelebung' des Metalls, gab es die ,Feuerluft' wieder ab, und zwar genau so viel, wie die Luft zunahm. Den Stoff nannte er das Oxygène, ,Sauerstoff‘. Was er auf der Basis exakten Messens und Wiegens dargestellt hatte, hieß später die ,Oxydation“ und die ,Reduktion' der Metalle.

Er analysierte Verbindungen und bestimmte ihre Gewichtsanteile, stellte die Chemie auf Maß, Zahl und Gewicht und inaugurierte zugleich eine international verbindliche, einheitliche, logisch durchsichtige Nomenklatur. Damit schuf er ein leicht zu handhabendes Instrument, um die sich auftürmende chemische Erfahrung zu ordnen und sprunghaft zu erweitern. Seine mit andern verfaßte Méthode de nomenclature chimique erschien 1787 und 1789 Lavoisiers Traité élémentaire de Chymie, das Grundbuch der modernen Chemie (vgl. Pörksen 1986b: 27f.). Die chemische Revolution war ausgearbeitet, ehe die politische einsetzte.

Was war geschehen? Stahl hatte neunzig Jahre zuvor Verbrennungsvorgänge unter einem einheitlichen Gesichtspunkt $\mathrm{zu}$ deuten versucht und dafür den Namen ,Phlogiston“ gefunden. Dies angenommene ,principium der Brennlichkeit‘ erwies sich als ein Trugbild, in dessen Verfolg die Wissenschaft mit Riesenschritten vorankam. Auch Lavoisier hing ihm lange an und machte erst in den 80er Jahren den Schnitt, erklärte das Phlogiston für nichtig und ersetzte die Leerstelle durch die Oxydationstheorie. Der Mohr hatte seine Schuldigkeit getan. Es war, wie wenn jemand einen Pfeil in Nachbars Garten schießt und nun, auf der Suche, eine ganz und gar andere Welt kennen lernt, die ihn Pfeil und Bogen vergessen lässt. Der Mohr konnte verbrannt werden.

Die Pariser Wirklichkeit des Jahres 1789 setzte dem Märchen die Krone auf. Es kam zur Verbrennung. Lavoisier arrangierte die Szene im Arsenal. Auf der Bühne erschien das aus brennbarem Stoff hergestellte ,Phlogiston', wurde vom ,Oxygène' schwerer Verbrechen beschuldigt und von Stahl als dem advocatus diaboli verteidigt. Aber das arme Phlogiston ward zum Feuertod verurteilt; die schöne Madame Lavoisier trat in antikischem Gewand als Opferpriesterin auf und verbrannte es (Speter 1929: 331; Lockemann 1950: 10). So geschehen im Jahr der Französischen Revolution, die Lavoisier fünf Jahre später, u.a. weil sich unter seiner Regie als Generalpächter während des Königtums angeblich der Tabak verschlechtert hatte, das Leben kostete (Dannemann 1903: 314).

Der Aufstieg und Fall des Phlogiston wird in verschiedenen Geschichten der Chemie so überliefert; ist nun aber dieses parabolische Märchen selbst ein Märchen? Ist es Fiktion? Im 20. Jahrhundert melden sich andere Stimmen zu Wort. Sie heben nicht nur die Leistung Stahls hervor, dass er die Verbrennungsvorgänge einheitlich deutete, sondern sehen im ,Phlogiston“ eine Vorahnung des modernen 
Begriffs der ,Reaktionswärme‘ und des ,Energie‘-Begriffs. Eduard Färber (1927: 123f.) erkennt im Phlogiston „eine energetische Auffassung vom Stoff, die so eigentlich dem zwanzigsten Jahrhundert anzugehören scheint. Die Phlogistik kann als ein Versuch in der Richtung auf eine Energetik hin angesehen werden.“ „Heute müssen wir hier einen Ansatz sehen, der aus einer Verstofflichung der Energie zu einer energetischen Theorie der Stoffe sich entwickeln wollte“.

Christa Habrich schlägt den umgekehrten Weg ein: sie versucht, auf eine für mich überzeugende Weise, den Begriff Stahls unabhängig von der Zukunft historisch genau zu lokalisieren.

Die Phlogistontheorie ist nämlich nicht der oft geschmähte Holzweg zur neuzeitlichen, wissenschaftlichen Chemie. Sie ist Stahls Versuch, neuplatonisch-alchimistische Deutungen für die der unbelebten Natur innewohnenden Bewegungen und Kräfte zu formulieren und die beobachteten Phänomene geistig zu bewältigen.

In der Tat kann mit einigem Recht das Phlogiston, das Stahl als das bewegende, die Verbrennung bewirkende Prinzip in den unbelebten Körpern beschreibt, in Analogie zur Anima der lebendigen Organismen interpretiert und verstanden werden, als die Kraft des ,principium movens‘, dem die Kraft der anima im ,motus vitalis‘ entspricht. Phlogiston und Anima sind immateriell. So wirkt es ganz verständlich, daß Stahl keine quantitativen Experimente im Sinne der Chemiker der Aufklärung zur Stützung seiner Phlogistonlehre anstellte, es wäre ihm ebenso absurd erschienen, einen Menschen vor und unmittelbar nach dem Tod zu wiegen, um durch Subtraktion das Gewicht der Seele zu errechnen! Das Phlogiston war ein naturphilosophischer Begriff und wurde erst im Laufe des 18. Jahrhunderts durch Stahls Anhänger [...] ,materialisiert‘ [...]. (Habrich 1985: 165)

Wie wurde die chemische Revolution in Deutschland um 1790 aufgenommen? Hermbstädt, der schon 1792 eine Übersetzung von Lavoisiers Traité élémentaire vorlegte, schreibt in der Vorrede der zweiten Auflage von 1803:

[...] damals war es ein Verbrechen, wenn man nicht den deutschen Heerführern des Phlogistons am Gängelbande nachfolgte; und man war ohne Rettung jeder Mißhandlung ausgesetzt, wenn man es wagen wollte selbst zu denken, ohne den Geist in Fesseln einzuzwängen. Ich fürchtete indessen nicht die Kreuzigung mit welcher mir gedrohet ward, meine Uebersetzung erschien auf deutschem Boden [...]. Jetzt, nach einem Zeitraume von 11 Jahren, hat sich alles geändert, selbst die eifrigsten Phlogistiker haben zur Fahne der Antiphlogistiker geschworen, und die Wissenschaft hat unendlich dabei gewonnen: ein Beweis daß die Wahrheit immer ihr Recht behauptet. (Lavoisier 1803: IIIf.)

Ist diese Darstellung zutreffend? Und wie stellte sich Lichtenberg $\mathrm{zu}$ der neuen antiphlogistischen Chemie? Als ich das Thema dieses Vortrags vorschlug, hatte ich gelesen, Lichtenberg habe die neue Chemie abgelehnt. In der Tat schreibt zum Beispiel Paul Hahn in seiner Arbeit Georg Christoph Lichtenberg und die exakten Wissenschaften: 
Lichtenbergs ablehnende Haltung gegen die Lavoisiersche Lehre ist damit deutlich gekennzeichnet als entstanden aus seinem Widerwillen gegen den aus einseitiger Spezialforschung folgenden unphilosophisch = schnell fertigen Aufbau neuer Systeme ohne Prüfung des alten Grundes. (Hahn 1927: 62)

Auch Dieter Herrmann (1969) betont die ablehnende Haltung. Das klang interessant; es erinnerte an den Fall eines anderen deutschen Autors, der sich der modernen mathematisch-quantitaiven Naturwissenschaft entgegenstellte. - Erlauben Sie daher eine Digression.

\section{2}

1795 erschien unter dem hoffnungsvollen Titel Exempel das folgende Distichon (WA I, 5: 229):

Schon ein Irrlicht sah ich verschwinden, dich, Phlogiston! Balde, O Newtonisch Gespenst! folgst du dem Brüderchen nach.

Der Parallelfall ist Ihnen bekannt. In Weimar erhob sich am Ende des 18. Jahrhunderts überraschend Widerstand gegen Newtons Optik. Der Augenblick, in dem dieser Widerstand sich bildete, ist von dem, der ihn leistete, aufs genaueste überliefert worden. Er hatte von Hofrat Büttner in Jena Prismen entliehen, um sich der optischen Fragen anzunehmen, hatte dann aber über anderen Arbeiten versäumt, Newtons Versuche nachzustellen. Der Hofrat erbat die Prismen zurück, sandte schließlich einen Boten und nun, sozusagen im letzten Augenblick, ergriff Goethe die Gelegenheit, durchs Prisma zu schauen:

Eben befand ich mich in einem völlig geweißten Zimmer, ich erwartete, als ich das Prisma vor die Augen nahm, eingedenk der Newtonischen Theorie, die ganze weiße Wand nach verschiedenen Stufen gefärbt, das von da in's Auge zurückkehrende Licht in soviel farbige Lichter zersplittert zu sehen.

Aber wie verwundert war ich, als die durch's Prisma angeschaute weiße Wand nach wie vor weiß blieb, daß nur da, wo ein Dunkles dran stieß, sich eine mehr oder weniger entschiedene Farbe zeigte, daß zuletzt die Fensterstöcke am allerlebhaftesten farbig erschienen, indessen am lichtgrauen Himmel draußen keine Spur von Färbung zu sehen war. Es bedurfte keiner langen Überlegung, so erkannte ich, daß eine Gränze nothwendig sei, um Farben hervorzubringen, und ich sprach wie durch einen Instinct sogleich vor mich laut aus, daß die Newtonische Lehre falsch sei. Nun war an keine Zurücksendung der Prismen mehr zu denken. (WA II, 4: 295f.) 
Der Fall ist nur allzu bekannt: Wie Goethe seit jenem Jahr 1791 mit seinen vielfältigen Versuchen auf dem Gebiet der Optik beginnt und seine ersten Ergebnisse den Freunden mitteilt, wie er sich Newton bald auch öffentlich mit zunehmender Deutlichkeit widersetzt und nun seinerseits Widerstand findet, wie alle Gemüter durch die Folgen der Französischen Revolution aufgeregt und die Physiker und Chemiker mit den Gasarten und dem Galvanismus beschäftigt sind (WA II, 4: 300), sich aber zu Lichtenberg ein beiderseits anregendes, fruchtbares Verhältnis herstellt, das dann allerdings abbricht, weil Lichtenberg ausweicht, schweigt, als er gedrängt wird, sich zu dem „ekelhaften Newtonschen Weiß“ zu äußern und nicht einmal die Freundlichkeit hat, die Goethe'schen Beiträge zur Optik in der letzten Ausgabe seines Erxleben zu erwähnen (WA II, 4: 302), wie nach weiteren zehn Jahren das große dreibändige Werk der Farbenlehre erscheint und zwar nicht echolos bleibt, aber doch eigentlich nur in Jena und Berlin Gefolgschaft findet: Im Ganzen ein über vier Jahrzehnte mit großer Hartnäckigkeit ausgetragener erfolgloser Kampf.

Der Gegensatz zwischen Newton und Goethe und die katalysatorhafte Gastrolle Lichtenbergs in diesem Stück ist von Heinwig Lang (1983) vor der Lichtenberggesellschaft genau und erhellend abgehandelt worden. - Hier interessiert dieser Fall als Parallele oder Kontrast zu dem Thema Lichtenberg und Lavoisier, Lichtenberg und die neue exakte Chemie, und ich beschränke mich auf eine Frage:

Wie erklärt sich Goethe den, wie er meinte, allem vernünftigen Augenschein widersprechenden Erfolg der Lehre Newtons? Ihre fortdauernde Geltung?

Der Grund lag seiner Überzeugung nach in der Trägheit der einmal geschaffenen Institution. Wissenschaftliche Lehren gelten so lange als wahr, wie ihre Urheber und deren Schüler am Leben sind und eine Lehrkanzel innehaben; von dieser Wahrheit war er durchdrungen. Er sah sich einer dünkelhaften Schule gegenüber: „[...] ich kannte damals, ob ich gleich alt genug war, die Beschränktheit der wissenschaftlichen Gilden noch nicht, diesen Handwerkssinn, der wohl etwas erhalten und fortpflanzen, aber nichts fördern kann [...]“ (WA II, 4: 304).

Die Situation des erfolglosen Außenseiters führte nicht nur zu einer so grundsätzlichen wissenschafts-soziologischen Erkenntnis: ihr Nebenprodukt war auch eine radikale Sprachkritik. Goethe beobachtete am Beispiel wissenschaftlicher Überlieferung die institutionalisierende Wirkung der Sprache. Er schätzte die Bedeutung der geprägten Sprache für die Tradierung geistiger Inhalte sehr hoch ein. Die Sprachprägung des Bewusstseins wird von ihm aber nicht als Prinzip behauptet, sondern beobachtet als historisches Faktum, das in der Neigung begründet ist, sich unter Autorität und Überlieferung zu beugen und das Wort für die Sache zu nehmen. Die Sprache erlaubt es, zwischen Subjekt und Objekt wahnhafte Zwischenwelten zu schieben. Newtons Spektrum war in diesem Sinn, 
was das Wort spectre vor Newton im Englischen bedeutet hat: ein Phantom, ein „Augengespenst“ (WA II, 4: 245).

Die Newton'sche Lehre erschien als eine gespensterhafte Vorstellungswelt, die sich gegenüber dem Gegenstandsbereich, auf den sie sich bezog, verselbständigt hatte und seit hundert Jahren als bloßer ,Wortkram‘ auf Kathedern und in Lehrbüchern überliefert wurde: eine Festung, von der man nur noch nicht bemerkt hatte, dass man es mit einem einsturzreifen „Ratten- und Eulennest“ zu tun hatte (LA I, 4: 5ff.).

Die unvermeidliche Begleiterscheinung einer institutionalisierten Schule und einer zugehörigen „Sprache, in der man sich nach seiner Art versteht“, ist aber: die Wahl einer unerwarteten Sprache führt zur Apperzeptionsverweigerung. Wer sich nicht an die auf einem Gebiet eingeführte Gattung hält, muss damit rechnen, gar nicht erst ernstgenommen zu werden. Goethe glaubte das bei der Aufnahme seiner botanischen Arbeiten beobachtet zu haben und fand es bestätigt, als seine Arbeiten zur Optik erschienen: „Hätte ich Chromatik gesagt, so wäre es unverfänglicher gewesen; denn da die Optik zum größten Teil mathematisch ist, so konnte und wollte niemand begreifen, wie einer der keine Ansprüche an Meßkunst machte, in der Optik wirken könne. “ Man verwunderte sich „höchlich, wie jemand, ohne höhere Einsicht in die Mathematik, wagen könne, Newton zu widersprechen. Denn daß eine Physik unabhängig von der Mathematik existiere, davon schien man keinen Begriff mehr zu haben. Die uralte Wahrheit, daß der Mathematiker sobald er in das Feld der Erfahrung tritt, so gut wie jeder andere dem Irrthum unterworfen sei, wollte niemand in diesem Falle anerkennen“ (WA II, 4: 304f.).

Eine solche Psychologie der Rezeption behält ihre Richtigkeit, selbst wenn ihr Anlass bestreitbar ist. Goethe bestärkte sie in der Hoffnung, das ,Spektrum“ Newtons werde verschwinden, wie kürzlich das ,Phlogiston“ verschwunden sei. Diese Kinder der letzten Jahrhundertwende waren, wie er meinte, beide in gleicher Weise geisterhaft:

Schon ein Irrlicht sah ich verschwinden, dich, Phlogiston! Balde, O Newtonisch Gespenst! folgst du dem Brüderchen nach.

\section{3}

Lichtenberg ist dem Drängen Goethes, sich zu dem ekelhaften ,Newtonschen Weiß` zu äußern, ausgewichen. Lehnte er seinerseits die neue antiphlogistische Chemie, den exakten Nachweis von Oxydation und Reduktion, wirklich ab? Es gibt darüber auch andere Auffassungen als die zitierte von Hahn. Sein Hörer 
Gottlieb Gamauf behauptet in seinen Erinnerungen aus Lichtenbergs Vorlesungen über Erxlebens Anfangsgründe der Naturlehre (1. Bändchen, Wien/Triest 1808), dass Lichtenberg der neuen Chemie „von ganzem Herzen ergeben war“ und führt dann ihre Vorzüge vor, darunter ihre vereinfachte und logisch aufgebaute Nomenklatur (Gamauf 1808: 537, 553ff.).

Das Buch von Georg Kahlbaum und August Hoffmann über Die Einführung der Lavoisier'schen Theorie im besonderen in Deutschland, das 1897 in Leipzig erschien, bezeichnet Lichtenberg einmal als „teilweisen Anhänger der neuen Lehre" und rechnet ihn dann ganz zu der recht großen Zahl deutscher Chemiker, die sich bis 1795 „für das Lavoisier'sche System direkt ausgesprochen haben“ (Kahlbaum \& Hoffmann 1897: 110f.). Das Buch hat allerdings einen apologetischen Zug (vgl. Kahlbaum \& Hoffmann 1897: 148f.); der Wissenschaftshistoriker Dannemann (1903: 313f.) fasst es 1903 zusammen: „Danach ist die Annahme, daß Deutschland sich länger als die übrigen Länder gegen die Annahme der Lehre Lavoisiers verschlossen habe, nicht gerechtfertigt.“ Das Prinzip der nationalen Konkurrenz, den Naturwissenschaften längst eingebaut, schlug sich auch in deren Überlieferung nieder; es wurde die Geschichte des Sieges der exakten Wissenschaften über die im Halbdunkel tappende Unwahrheit, und diese Geschichte fragte: war er auf unsrer Seite, lag Lichtenberg bei dem Rennen der Nationen in die Modernität früh vorn?

Wie also stand er zur neuen Chemie? Es gibt darüber nur einige aufschlussreiche Seiten in der erwähnten Abhandlung von Paul Hahn aus dem Jahr 1927 und in der Abhandlung Dieter Herrmanns von 1969.

Ad fontes! Und dies mit einer captatio benevolentiae: Das Folgende ist nur eine Skizze, sehr vorläufig, von jemandem, der weder Physiker noch Chemiker ist und der bisher noch nicht alle durchgearbeiteten Stellen zu verstehen glaubt.

Es wäre sonderbar, wenn Lichtenberg aus der chemischen Revolution keine Funken geschlagen hätte. Ich gebe sieben Beispiele, unsystematisch, und nenne ihre Themen:

\section{Das Janusgesicht des Fortschritts}

Am $1^{\text {ten }}$ August 1774 entdeckte Priestley bekanntlich die dephlogistisierte Luft. Man nannte diesen Tag den Geburtstag der antiphlogistischen Chemie. Am $1^{\text {ten }}$ August 1759 wurden die Franzosen zu Land bei Minden, und am $1^{\text {ten }}$ August 1798 zur See bei Abukir geschlagen. Sind das Geburts- oder Sterbe-Tage? (L 676).

Die Frage könnte über der Geschichte der neuzeitlichen Naturwissenschaft stehen. Dem Märchen vom Fortschritt und von der Enthüllung der Wahrheit durch die Wissenschaft wird durch zwei Züge Schach geboten: es wird mit der Geschich- 
te der Kriege in Parallele gesetzt, also mit einem Feld, auf dem primär um Macht gerungen wird und wo Sieg oder Niederlage zwei Namen für dieselbe Sache sind; und die Geschichte der Siege wird durch die der Verluste in Frage gestellt. - Der Geburtstag der neuen Unternehmer-Chemie war ja in der Tat der Sterbetag einer 3000 Jahre alten Handwerker-Chymie.

\section{Die Erwartung eines Paradigmenwechsels in der Chemie}

Ich kann eben nicht sagen, daß mir diese Entdeckungen von neuen Erden sehr gefallen. Diese Aufhaufungen von neuen Körpern erinnern mich an die Epicykloiden in der Astronomie. Was wollten jene Astronomen mit ihren Epicykloiden gemacht haben, wenn sie die Aberration der Fixsterne hätten sehen können. Viel geometrischer Scharfsinn hätte können gezeigt werden, wie z. Beispiel Kopernikus bei sein[en] Irrtümern. Aber was ist das? - Was ich eigentlich hier sagen wollte ist: Wenn die Chemie nicht bald einen Kepler erhält, so wird sie von der Menge von Epicykloiden erdrückt werden; kein Mensch wird sie mehr studieren, und die Trägheit wird sie am Ende zu simplifizieren wissen, was der tätige Verstand besser könnte. Es muß und muß einen Standpunkt geben, aus welchem angesehn, alles einfacher aussieht. (L 962)

Lichtenberg erwartet einen Paradigmenwechsel in der Chemie; das Fach ist unter einen zunehmenden „Erfahrungsdruck“ (Lepenies 1976: 21ff., 63 u. ö.) geraten und nicht mehr in der Lage, auf der bisherigen Grundlage die immer weiter sich ausdifferenzierenden und verkomplizierenden empirischen Befunde $z u$ ordnen und übersichtlich zu beschreiben. Es befindet sich in einer Krise. Die Situation erinnert an die der Astronomie vor Kepler, der die Beschreibung der Planetenbahnen durch die Idee, sie als Ellipsen aufzufassen, mit einem Schlage vereinfachte. - Kurz: Lichtenberg entwirft hier modellhaft in einem Aperçu jene Struktur naturwissenschaftlicher Revolutionen, durch deren Aufstellung Thomas S. Kuhn 1962 weltberühmt geworden ist. Auch Kuhn (1967: 100ff., 122, 160; vgl. auch Gipper 1978) hatte die Krisensituation vor Lavoisier und Kepler verglichen.

\section{Streit, Spruch und Widerspruch als Voraussetzung der Erkenntnis}

Madame Lavoisier als Priesterin angekleidet verbrannte feierlich das Phlogiston in einer Versammlung. Hierbey läßt sich nichts weiter sagen als: Wäre Newton im Stande gewesen durch seine Frau, wenn er eine gehabt hätte, die Cartesianischen Wirbel verbrennen zu lassen; so hätte er unmöglich seine principia schreiben können. Man sieht der Fund selbst war den Erfindern unerwartet. (Erxleben 1794, Vorrede zur 6. Aufl.: XXIII) 
Mit anderen Worten: Das Oxygène verdankt seine Existenz dem Phlogiston. Lavoisiers Triumph erweist sich als „klein“ und „kindisch“; er wäre im Fall Newtons undenkbar. - Descartes dachte sich die Planeten in kreisenden Ätherströmen schwimmend, deren Mittelpunkt die Sonne bilden sollte. Eine Wirkung der Himmelskörper in die Ferne schien den Anhängern dieser die Astronomie vor Newton beherrschenden ,Wirbeltheorie“ unannehmbar. Newton setzte dem das Gesetz der nah wie fern wirkenden Schwerkraft entgegen, das Gravitationsgesetz, und formulierte im Gegenzug zu Descartes: „Daß durch die Zentralkräfte die Planeten in ihren Bahnen erhalten werden können, ersieht man aus der Bewegung der Wurfgeschosse“ (Dannemann 1903: 203).

Die neue Physik verdankt sich dem Widerspruch zu Descartes, wie das Oxygène seine Existenz dem Phlogiston schuldet. War Lavoisiers Erfolg vielleicht ein zufälliger Glücksfall? Lichtenberg setzt auf das Streitgespräch. Schon am Anfang der Vorrede zur sechsten Auflage von Erxleben (1794: XXI) heißt es: „Ganz davon [von der neuen Chemie] zu schweigen wäre unverzeihlich gewesen. Eine Lehre, die so vielen Beifall erhalten hat, und täglich noch mehr erhält, verdient wenigstens Respekt selbst von dem, der sich noch nicht ohne Einschränkung dazu bekennt. Untersuchung der Natur ist durch den Streit darüber befördert worden, und mehr, als durch irgend einen andern über Lehren der Physik in diesem Jahrhundert. Und was kann der aufrichtige Beförderer der Naturlehre Größeres wünschen! Wenn nur untersucht wird, die Triebfeder dabey möge seyn was sie wolle.“

Die bisher zitierten Stellen werfen Schlaglichter auf eine mögliche verbesserte Wissenschaftsgeschichte.

\section{Der Übersprung von der naturwissenschaftlichen Erkenntnis ins Alltagsweltbild}

In unsrer Gegenwart hinkt das Alltagsweltbild weit hinter dem der Naturwissenschaften her. Diese Kluft war im 18. und 19. Jahrhundert allgemein noch nicht so tief, Lichtenberg verfügte aber über eine exzeptionelle Fähigkeit, sie durch die Art seiner wissenschaftlichen Darstellung und durch seine die alltägliche Erfahrungswelt imaginierenden Gedankenexperimente zu überspringen. Wir finden Beispiele im Erxleben (1794); sie erläutern sich selbst:

§ 480: Besteht nun vielleicht die Hitze oder Wärme in nichts anderm, als in einer zitternden Bewegung der Theilchen, woraus ein Körper gebauet ist? Dann muß sich aber diese Bewegung nur auf die allerfeinsten Theilchen des Körpers erstrecken, die so zart sind, daß sie ihre Bewegung den Lufttheilchen nicht mittheilen können, denn sonst würde ein Schall davon entstehen $(\S 246)$. 
§ 481: Giebt es also etwa vielmehr eine eigene Materie des Feuers, ein Elementarfeuer, ein sehr feines flüssiges Wesen, das durch die Zwischenräume aller Körper gleichförmig ausgebreitet ist, und in dessen Zittern die Wärme besteht?“ (418f.)

$\S 494 \mathrm{~V}$ : Unsere Luft ist ein Feuer-Meer, das die Erde zersetzen könnte, wenn sie zersetzt würde. Überall kann Wärme entstehen wo Capacitäten verändert werden, oder gebundene Wärme frey wird. Die Wärme in der Erde, dieser großen chemischen Werkstätte, zu erklären brauchen wir kein Centralfeuer, so wenig als in der Tonne voll gärenden Mostes [...]. (452)

\section{Aus den Briefen:}

Wenn wir ohne Wärme leben könnten und einige Meilen über der Erde wohnten, so würden wir die Sonne nicht Feuer sondern Licht genannt haben. (An Wolff, 12.9.1782)

Großer Gott! Wenn die Welt statt unsrer jetzigen Luft mit dephlogistisierter umgeben wäre. Ich glaube, man könnte die Welt mit einer Pfeife Tabak in Brand stecken. Gera wäre gewiß in 2 Minuten angebrannt. (An Wolff, 30.6.1782)

\section{Erkenntnis als Entwurf. Die Theorie der Vorstellungsarten und der Bildersprachen}

Lichtenberg verbindet mit Goethe eine gründliche Erkenntnis- und Sprachskepsis. In der Vorrede zur 3. Auflage des Erxleben heißt es über die Lehre von den zwei elektrischen Fluida:

Ich sehe solche Hypothesen in der Physik für nichts weiter an als bequeme Bilder, sich die Vorstellung des Ganzen zu erleichtern. Die Vorstellungsart, die die größte Erleichterung gewährt, ist die beste, soweit sie auch von der Wahrheit selbst, der wir uns dadurch zu nähern suchen, entfernt seyn mag. (XXV)

In der 6. Auflage (Erxleben 1794) heißt es in verwandtem Zusammenhang:

Freylich wird von der eigentlichen Natur des Feuers immer noch vieles vor unsern Augen verborgen bleiben, allein wenn auch alle diese Vorstellung=Arten von der absoluten Wahrheit sehr weit entfernt bleiben, so haben sie doch immer für uns einen sehr großen relativen Werth, sie sind schickliche Bilder uns die mannichfaltigen Erscheinungen darunter im Zusammenhang zu denken und uns die Kenntnis derselben zu erleichtern. Gesetzt die Ursache der Hitze sey kein Fluidum, es sey etwas wovon sich nichts Gleiches in der Natur fände, so ist es doch nicht zu leugnen, daß sich die Erscheinungen, so weit wir sie kennen, sehr schicklich unter dem Bilde eines flüssigen Wesens denken lassen, und ist ein solches Zeichen glücklich gewählt, so kann es selbst dienen den Geist auf neue Verhältnisse des unbekannten Wesens zu leiten.

Lichtenberg gibt dafür Beispiele und fährt dann fort: 


\begin{abstract}
Was Wunder also wenn jene Männer anfangen ihre Erklärungen der natürlichen Phänomene für etwas mehr als bloße Bilder=Sprache zu halten - Und was ist dann das reelle in unsern Vorstellungen von Dingen außer uns überhaupt, und was haben sie für Verhältnisse zu denselben? Laßt uns daher immer jene Bilder Sprache studiren und uns bemühen ihr mehr Reichthum zu geben, so treffen wir am Ende vielleicht die Wahrheit so, wie sie der unterrichtete Taubstumme endlich trifft, der unsere Sprache für das Ohr, für eine für das Auge, und was eigentlich Töne sind, für Bewegung der Kehle und der Lippen hält, aber indem er sich die letztere zu sprechen bestrebt, auch demjenigen Sinne, ohne es zu wissen, vernehmlich spricht, dessen er gänzlich beraubt ist. L. (§ 494. Z., 453f.)
\end{abstract}

Diese Theorie der Vorstellungsarten berührt sich aufs engste mit Goethes etwa gleichzeitiger naturwissenschaftlicher Erkenntnislehre und fußt wie diese auf Kant. ${ }^{2}$ Auch für Goethe sind Hypothesen wie die beiden konkurrierenden biologischen Theorien des 18. Jahrhunderts ,Epigenese“ und ,Evolution', oder auch die des ,Atomismus' und ,Dynamismus', Vorstellungsarten. Und die ihnen jeweils entsprechenden Termini und Terminologietypen sind in dem gleichen Sinn perspektivisch gebundene Erfassungsversuche, Bildersprachen, von dem Gegenstand selbst durch eine Kluft getrennt. Sie können, so meinen beide, schicklich und glücklich gewählt sein: der eine Schlüssel mag nach Goethe „bequemer“ zu brauchen sein als der andere. Lichtenberg rät, sie zu studieren und ihnen mehr „Reichtum“ zu geben - Goethe empfiehlt, sich ihrer „mit Bewußtsein“ zu bedienen und sie zu variieren, die Perspektiven zu bündeln: dann könne, meinen beide, eine weitere Annäherung an die Wahrheit erfolgen. Die Erkenntnis könnte dann laut Lichtenberg ihr Suchgerät, die vorausgeworfene Metaphorik, fast einholen: sie bleibe freilich von der Sache selbst immer noch getrennt. Er gibt hier ein singuläres, hinreißendes Bild dafür, wie die Sprache dem Gegenstand isomorph sein kann und zugleich bloßer Widerschein bleiben muss: so wie die Sprache des Taubstummen, die selbst im Medium und Horizont der Nachahmung von äußerlichen Sprechbewegungen verharrt und zugleich doch vernehmlich wird.

\title{
6 Kritik an erklärenden, orientierenden Termini der neuen Chemie
}

Anfang 1792 äußert sich Lichtenberg über Zweifel an der französischen Chemie:

2 Vgl. Kleinschnieder 1971; Zehe 1986. Vgl. zum Folgenden in Pörksen 1986a die Kapitel „Wissenschaftssprache und Sprachauffassung bei Linné und Goethe“ (72-96) und „Goethes Kritik naturwissenschaftlicher Metaphorik und der Roman ,Die Wahlverwandtschaften““ (97-115, bes. 97110). 
Diese schaden ihrer jetzigen Vorzüglichkeit nicht, machen mir aber die Nomenklatur im Ganzen verächtlich. Hypothesen sind Vota, aber neue Namen sind Decreta, wo will das am Ende hinaus? Die neue Chemie wird gewiß nicht dauern [...]. Die Namen aber werden bleiben, und müssen gelernt werden, solange noch vortrefflich Sachen damit vorgetragen werden. (an [?] Bw 3, Nr. 2033).

Er erwartet also von der Sprache der neuen Chemie eine Verselbständigung und Fortdauer durch einen Überhang der Institution, wie Goethe sie am ,Phlogiston“ und am ,Spektrum' zu beobachten glaubte. Lichtenbergs Kritik betrifft nicht die neue Nomenklatur insgesamt, die in der Regel übrigens als große Leistung hervorgehoben wird. In der Vorrede der 6. Auflage des Erxleben (1794) äußert er sich ausführlich zu dem Thema (XXXV-XLI), parallel dazu und konzentrierter in den Sudelbüchern. Er lobt das System der Nachsilben, das die Typen der Sulfate, Sulfite und Sulfure wie die Fälle einer Deklination zu unterscheiden erlaubt, als eine ,herrliche Methode“ (Erxleben 1794: XXXVf.), kritisiert aber die neuen Termini vom Typ ,Oxygène‘. Oxygène ist ein durchsichtiger, sprechender, erklärender, orientierender Ausdruck, kein Name. Er spricht aus, dass der gemeinte Stoff säuert, ist also ein Prädikat. Lichtenberg spricht von einer ,Hypothese‘. Angesichts der grundsätzlichen Möglichkeit, zwischen dem orientierenden, definierenden Terminus und dem Zeichenterminus zu wählen, plädiert er entschieden für den Zeichenterminus. „Die Wörter sollen ja bloße Zeichen für den Begriff und keine Definitionen seyn“ (Erxleben 1794: XXXVI). Aus demselben Grund tritt er dafür ein, einmal eingeführte Termini beizubehalten.

Seine Überlegungen zu dieser grundlegenden Frage an die Wissenschaftssprache sind von größtem Interesse, auch wenn ihr Anlass fraglich sein mag. Sein Plädoyer für den Zeichenterminus und für den eingeführten, zur Konvention gewordenen Terminus hat folgende Gründe:

a) Ein Zeichenterminus ist auch dann weiter verwendbar, wenn der durch ihn bezeichnete Begriff sich wandelt, was in der Geschichte der Wissenschaft regelmäßig eintritt. Der Name funktioniert weiter als Etikett für die sich wandelnde Sache.

b) Der Inaugurator neuer orientierender Termini hat etwas von einem Eroberer, der Städte und Straßen umbenennt; er dekretiert Hypothesen und versucht, sie festzuschreiben. Sauerstoff, Oxygène, und seine Ableitungen beruhen vorerst auf Annahme.

Wer Hypothesen schafft, gibt bloß sein unmaßgebliches Gutachten, und das ist niemandem verwehrt, wer sie der Sprache aufzwingt, publicirt Mandate, und da gehört schon was dazu sie durchzusetzen. (Erxleben 1794: XXXIX)

c) Orientierende Termini sind zudem immer nur teildefinierend, sie erklären ihren Gegenstand nie vollständig, oft nur einen geringen Teil; 
d) und sie werden sehr rasch in Zeichentermini umgedeutet. Um es linguistisch auszudrücken: Ihr Etymon verblasst, sie unterliegen einer Idiomatisierung, Lexikalisierung und stehen dann bald als erweitert gebrauchte selbständige Zeichen da. Auch in der Mathematik, die ohne Hypothesen arbeitet und daher ein Recht auf definierende Termini hätte, unterliegen die ursprünglich durchsichtigen Ausdrücke ,Ellipse‘, ,Parabel‘, ,Hyperbel‘ einer solchen Lexikalisierung. Niemand denkt an ihre Ursprungsbedeutung. Was Karl Bühler (1965: 349) die „Sachsteuerung des Verstehens“ nannte, gewinnt die Oberhand.

\begin{abstract}
Man ist daher, wie mich dünkt, viel zu ängstlich, mit der Abschaffung von sehr gangbaren Worten, die den Gegenstand, den sie bezeichnen, unrichtig erklären, gewesen. Das konnte schlechterdings nicht schaden, da man es schon in tausenden von Fällen gewohnt ist keine Erklärung in den Wörtern zu suchen, sondern blos ein Zeichen für den Begriff mit welchen man sich sonstwo bekannt gemacht hat. So hätte das Wort Metallkalch, als allgemein bekannt, gar wohl auch können beybehalten werden, da in ganz Europa niemand mehr dabey an Kalcherde dachte. (Erxleben 1794: XXXVII)
\end{abstract}

e) Der Wert eines Worts beruht in erster Linie auf Konvention, auf „Popularität oder Gangbarkeit“ nach Lichtenbergs Ausdruck.

\begin{abstract}
Hypothesen zu machen und sie als seine Stimme der Welt vorzulegen, darf niemand gewehrt sein, sie gehört dem Verfasser, aber die Sprache gehört der Nation und mit dieser darf man nicht umspringen wie man will. Die Gründe diese zu ändern müssen sehr triftig sein und eine neue Hypothese ist nie eine triftige Ursache, wie die Geschichte der Physik hinlänglich lehrt. Finden solche Wörter Eingang so vergißt man bald was sie sagen sollen, und denkt nur an die Sache, die sie bezeichnen, nach ihrem ganzen Umfang, und dieses ist die glücklichste, wenigstens die unschädlichste Periode solcher Benennungen. (Erxleben 1794: XXXVIII)
\end{abstract}

f) Aus diesen Gründen dürfen „falsche“ orientierende Termini nicht überschätzt werden und haben undurchsichtige Namen, arabische, Fremdwort-Termini überhaupt, keinen Nachteil. Die Geschichte der Astronomie erscheint als Beleg; weder ihre falschen noch ihre unverständlichen Wortzeichen haben den Fortschritt der Wissenschaft aufgehalten (Erxleben 1794: XXXIXf.).

g) Gibt man die alten Namen auf, so versteht man die alten Schriften nicht mehr.

Das Fazit: Lichtenberg schlägt vor, wenn man eine neue Terminologie einführt, dies auf philosophischer Basis zu tun. Diese Basis legt nahe, keine neue Terminologie einzuführen, wo es nicht notwendig ist, und wo es nötig wird, der neuen Kunstsprache keine Hypothesen beizumengen, sondern sich arbiträrer Zeichentermini zu bedienen. Ein ungewöhnlich klares Konzept! 


\section{Die Stellungnahme: Suche nach einer einheitlichen Erklärung}

Wenn wir auf die bisherigen Abschnitte zurückschauen - 1. Das Janusgesicht des Fortschritts, 2. Die Erwartung eines Paradigmenwechsels in der Chemie, 3. Streit als Voraussetzung der Erkenntnis, 4. Das Hin- und Herspringen zwischen Naturwissenschaft und Alltagserfahrung, 5. Erkenntnis als Entwurf. Die Theorie der Vorstellungsarten und Bildersprachen, 6. Kritische Überlegungen zur neuen chemischen Terminologie - wenn wir diese Antworten Lichtenbergs auf die Herausforderung durch die neue Chemie überschauen, entdecken wir Einsichten, die Wissenschaftsgeschichte und Wissenschaftstheorie verbessern könnten.

Seine Aufnahme der neuen Chemie ist offen und undogmatisch gewesen, skeptisch und gesellig; beim Spaziergang solle man sich zur Gesellschaft halten und sie nicht von weit her zurückrufen wollen, meint er. Den prinzipiellen Schritt zur quantitativen Chemie hat er nicht vollzogen. - Im Focus seines Interesses bleiben die Phänomene ,Licht‘, ,Wärme‘, ,Elektrizität‘, und er fragt nach ihrem Anteil an den chemischen Prozessen.

Er verkennt nicht die Schlüssigkeit des neuen Gebäudes, vermag es aber vorläufig nicht mit seinen physikalischen Vorstellungen $\mathrm{zu}$ verbinden: eine einheitliche Erklärung aber ist, was er sucht. „Es gibt eigentlich in sich selbst und vor Gott nur Eine Natur, der Mensch macht sich Capitel daraus, und tracktirt jedes besonders, als wenn jedes eine eigene Welt wäre“, schreibt er am 6. Januar 1785 an Wolff und wiederholt es fast wörtlich in der zitierten Vorrede zu Erxleben von 1794 (XXXIV):

Wenn ich die Franz. Chemie ein Meisterstück genannt habe; so will ich dieses bloß von ihr als einer isolirten Sammlung von Kenntnissen, oder wenn man will, von isolirter Wissenschaft, verstanden wissen, und nicht in so fern sie ein Theil der Naturlehre im allgemeinsten Verstande ist, die auch schon ihre festen Puncte hat oder zu haben glaubt, von denen sie ausgeht, so gut als diese Chemie. Wenn dereinst, alle die einzelnen bearbeiteten Theile der gesamten Naturlehre zu einem Ganzen zusammen gefügt werden sollen, so wird es sich finden welches die festesten Puncte von beyden gewesen sind. Vielleicht müssen alle etwas nachgeben um die schicklichsten Fugen zu treffen.

Das ist ganz im Sinne der Theorie der Vorstellungsarten, des relativen Rechts verschiedener Perspektiven und ihrer Bündelung gedacht.

Dieses ist dünkt mich das eigentliche Geschäfte des allgemeinen Naturforschers; die isolirten Beschäftigungen einzelner Classen zu vergleichen und zusammen zu nehmen. Denn wenn der Mensch hierin ja zu einem sichern Zweck gelangt: so muß alles Eins seyn. Führt diese Zusammenhaltung auf Zweifel gegen die Richtigkeit manches Einzelnen, so will man damit die Bemühungen hierzu nicht sogleich verwerfen, ja nicht einmal stöhren. Ein jeder thue das Seinige so gelangt man am geschwindesten zum Zweck. (Erxleben 1794: XXIVf.) 
Lichtenbergs Zweifel rühren von seinem Blickwinkel her, von der Elektrizität; in deren Chemie vermutet er, wie er Anfang 1792 in einem Brief schreibt, den zersprengenden Keim: „Die Zeit da die Chemie der Elektrizität entwickelt werden wird, wird vermutlich die sein, da die Franz. Chemie über den Haufen fallen wird.“ (Bw 3, Nr. 2033)

Die Prognose trifft nur insofern zu, als die Lehre von der elektrischen Ladung im atomaren Bereich tatsächlich entscheidende Aufschlüsse über chemische Verbindungen und Zersetzungen bereithielt: die quantitative Chemie fügte sich der Atomphysik aber ein und wurde von ihr nicht über den Haufen geworfen. Unsere Quellen zeigen freilich auch, daß die Frage, ob Lichtenberg auf Seiten der alten oder der neuen Chemie, der Sieger oder der Verlierer stand, in seinem Fall keinen Sinn gibt: er sprach von Dingen und war kein Dogmatiker. Und diese Frage gehört in die Märchentonne neuzeitlicher Naturwissenschaft, in der sich das Dogma der Sieger und die Vorstellung eines internationalen Pferderennens in die Modernität auf anmutige Weise vermischen.

Vielleicht ist ein Wort William Blakes besser geeignet, die Erwiderungen Lichtenbergs zu umreißen: Energy is eternal delight. 